\title{
On the Space-Time Interpretation of Classical Canonical Systems I: The General Theory
}

\author{
K. Drühl \\ Max-Planck-Institut zur Erforschung der Lebensbedingungen der wissenschaftlich-technischen Welt, \\ D-8130 Starnberg, Federal Republic of Germany
}

\begin{abstract}
We define a canonical system as a canonical manifold $M$ plus a canonical vectorfield on $M$. For such systems a unique kinematical interpretation is deduced from a set of Kinematical Axioms satisfied by the algebra of differentiable functions on $M$. This algebra is required to contain a subalgebra which is maximal commutative under the Poisson bracket. $M$ is shown to be diffeomorphic to the cotangent bundle over its quotient manifold, which is defined by the given subalgebra. Canonical systems satisfying these axioms are then classified. If the "phase space interpretation" is adopted they are shown to describe the motion of masspoints in some configuration space under the influence of and interacting by arbitrary vector and scalar potentials.
\end{abstract}

\section{Introduction}

In this paper we study the space-time interpretation of classical canonical systems. At a first glance this would seem to pose no problem. After all classical physics is concerned with phenomena occuring in space and time such as the motion of material bodies and their relation to the classical fields. Thus the basic theoretical quantities and dynamical principles are formulated in terms of space-time concepts, and no additional interpretation is required.

However a different situation is met if we wish to consider a classical theory as the limiting case of some corresponding quantum theory. In this case the space-time interpretation of the classical theory should itself be obtainable from some elements of structure which are already present in the quantum theory. Let us consider the algebraic formulation of quantum theory [1]. In this formulation operations corresponding to measurements are represented by elements in some non-commutative $*$-algebra over the complex numbers. The possible states of the physical systems under consideration are represented by positive linear functionals on the given algebra, which assign real numbers as "expectation values" to those elements which represent measurements. The 
traditional formulation of quantum mechanics is a special case where measurements are represented by self adjoint operators on some given Hilbert space.

A general framework like this does not contain any reference to spacetime concepts. In fact one may say that the main differences among the various known approaches to the formulation of relativistic quantum theories lie precisely in the way in which such concepts are incorporated. The most important and common feature in all quantum theories known to us, relativistic or non-relativistic, is the existence of some distinguished finite dimensional symmetry group, operating as a group of transformations on the classical space-time. This group (the Poincaré group in the special relativistic case) is postulated to operate as a group of automorphisms on the quantum theoretical structure in such a way that each automorphism corresponds in a unique way to the change in the observational situation as described by the appropriate transformation on the classical space-time [2].

This approach is well founded for non-relativistic and special relativistic theories. It may however meet with difficulties in the general relativistic situation, where no exact symmetry group can be expected to exist (see our discussion of symmetries in [11]). Of course the construction of such general quantum theories is still an unsolved problem. However similar problems may be expected to occur on the purely classical level, if we avoid any direct reference to the classical space-time manifold in our basic concepts. We shall therefore reanalyze the space-time interpretation of classical theories within a mathematical framework showing closer structural similarities to existing quantum theories.

One of the important features we wish to take over from the algebraic approach is the clear distinction between the two basic concepts of observables and states. The former represent operations of measurement, and we shall take the structure of the set of observables as the starting point of our analysis. The notion of states appropriate for the different classes of physical systems will then be introduced as a second step.

There is a well known formulation of classical particle mechanics incorporating this feature, namely the canonical one. In this formulation the observables are represented by continuous functions on the classical phase space $\Gamma$ while states correspond to measures on $\Gamma$. Expectation values are obtained by integrating the observables by the corresponding measures over all of $\Gamma$. If we restrict our attention to real valued differentiable functions which we shall call variables henceforth, the resulting set is a commutative algebra under pointwise multiplication, and a Lie-algebra under the Poisson-bracket [3]. This formulation is the starting point for any "canonical quantization" procedure of constructing quantum theories. On the other hand it is the formulation arrived at in the classical limit of non relativistic quantum theories.

The canonical approach is sometimes believed to meet with difficulties in the context of general relativity. Leaving aside the problem of a canonical formulation for the classical fields, these difficulties are traced to the non-existence of a unique time parameter.

In particular this makes it necessary to extend the phase space by the introduction of time-like variables. We shall however find that a straightforward axiomatic approach to the question of position and time-like observables proves most successful within our framework. The resulting theory applies equally 
well to non-relativistic, special relativistic and general relativistic situations. In addition it will make transparent important algebraic features of general relativity, which show up less clearly in the usual space-time tensorfield calculus. These may provide valuable hints to the structure of a possible general relativistic quantum theory. For relativistic canonical systems in particular see [11].

The basic object we shall study is the classical algebra of variables $\mathfrak{A}$, consisting of all real-valued differentiable functions on some fixed and given canonical manifold $M$ (for basic definitions and theorems see the Appendix). We wish to emphasize two points here. The manifold plays an auxiliary role only in carrying the algebra $\mathfrak{A}^{1}$. Apart from this no assumption is made about $M$, and no physical interpretation is given to it. In particular we do not assume or imply that $M$ be the phase space of some classical system.

The second point concerns our notion of observables, which is an extremely geometrical one. From our point of view any classical observation may be completely reduced to the observation of events in space-time and the observation of relations between infinitesimally close pairs of events (elements of direction). The precise statement of this will be a set of axioms concerning the structure of $\mathfrak{A}$.

Any such set of axioms must necessarily involve some aspects of the dynamics of the system like those related to the free fall of material points and the propagation of light rays ${ }^{2}$. These kinematical aspects are indispensable for any classical analysis of observations. For example, a time-like element of direction is nothing but an element which may occur on the path of some material point, and this condition is a dynamical one.

There are other aspects of the dynamics, such as the disintegration of particles or thermodynamic properties of bodies, which one may call internal. Such aspects refer to the properties of possible states on the classical algebra $\mathfrak{A}$, which will only be briefly treated in this paper.

In Chapter 2 we define a canonical system as an ordered triplet $(M, \Omega, Y)$, where $M$ is a manifold, $\Omega$ is a nowhere degenerate closed 2-form on $M$, and $Y$ is a vectorfield leaving $\Omega$ invariant ${ }^{3}$. In accordance with the remarks above $Y$ is meant to represent the kinematical aspects of the dynamics only. The algebra $\mathfrak{A}$

\footnotetext{
1 By considering all complex valued continuous functions vanishing at infinity on $M$ we obtain a commutative $C^{*}$-algebra, containing $\mathfrak{U}$ as a dense subset. On the other hand any commutative $C^{*}$ algebra is isomorphic to the algebra of continuous functions on the locally compact Hausdorff space $M^{*}$ of its maximal ideals ([7], see the generalization mentioned in $\S 16.3$ ). One may hope to even introduce a differentiable structure on $M^{*}$ by considering a suitable dense subalgebra, and hence arrive at a complete algebraic characterization of the underlying manifold. This would render the the introduction of $M$ superfluous. Although this aspect is not central for our present investigation, we would like to encourage any attempts in this direction

2 A careful analysis of these aspects has recently been given by Ehlers and others [4]. Using elementary operations with freely falling bodies and light rays as a starting point they were able to show that the differentiable, affine and Riemannian structure of space-time may be completely recovered from such operations. In accordance with these results we find that in our framework the dynamics of the physical system is to a large extent determined by those axioms which are necessary for constructing a space-time interpretation

3 Our definition of canonical systems differs from that given in [5], where the dynamics is introduced by means of a constraint submanifold. In the relativistic case this would exclude the possibility to treat states containing different masses, which is easily done in our framework (see [11])
} 
of variables on $M$ is required to satisfy a set of Kinematical Axioms. These Axioms postulate the existence of essentially two disjoint classes of variables, generating all other variables. The first class is a subalgebra $\mathfrak{U}_{0}$ which is maximal commutative with respect to the Poisson bracket; the second class is the linear subspace $Y\left(\mathfrak{U}_{0}\right)$.

The canonical vectorfields defined by functions in $\mathfrak{U}_{0}$ determine a set of maximal integral submanifolds. This set is shown to admit a unique differentiable structure by $\mathfrak{A}_{0}$; the resulting manifold is called the quotient of $M$. The functions in $Y\left(\mathfrak{U}_{0}\right)$ are shown to generate vectorfields on the quotient and may then be considered as the corresponding momenta. The main result is the Isomorphism Theorem 2.2 which states that there is a unique diffeomorphism of the original manifold $M$ to the cotangent bundle $L^{*}$ over its quotient $N$.

In Chapter 3 we give a complete classification of canonical systems in terms of their canonical forms and kinematical vectorfields. If we introduce states on $\mathfrak{A}$ as linear functionals defined by measures on $M$ and assume that the dynamics are completely specified by the vectorfield $Y$, a broad class of physical systems is obtained. This class describes the motion of masspoints in some configuration space of arbitrary pseudo Riemannian or Riemannian structure under the influence of arbitrary scalar and vector potentials.

A detailled treatment of relativistic canonical systems will be given in a subsequent paper [11]. They will be singled out by an additional set of Relativistic Axioms and shown to admit a unique space-time interpretation.

\section{Canonical Systems and Their Quotients}

For the sake of simplicity we shall only consider manifolds of class $C^{\infty}$. Some of the proofs in this chapter and the main theorems required for them may be found in the Appendix.

Definition 2.1. A canonical system is an ordered set $(M, \Omega, Y)$ where

i) $M$ is a manifold;

ii) $\Omega$ is a closed, nowhere degenerate differential 2-form on $M$;

iii) $Y$ is a vectorfield on $M$ leaving $\Omega$ invariant ${ }^{4}$.

The pair $(M, \Omega)$ is called a canonical manifold [3]. The canonical form $\Omega$ defines a bijective map from the space $\mathscr{L}$ of vectorfields on $M$ to the dual space of differential 1-forms. From the inverse map $\xi$ we obtain an anti-symmetric tensorfield $\Lambda$, which defines the Poisson bracket for differentiable functions. Vectorfields leaving $\Omega$ invariant are called canonical. They are exactly those for which the corresponding 1-form is closed, and satisfy the commutation relations below:

i) $\Omega\left(X_{1}, X\right)=\omega\left(X_{1}\right)=\omega\left(\xi_{\omega_{1}}\right)=\Lambda\left(\omega, \omega_{1}\right)$.

ii) $\left[\xi_{\omega}, \xi_{\omega_{1}}\right]=\xi_{d \Lambda\left(\omega, \omega_{1}\right)}$ for $d \omega=d \omega_{1}=0$.

i) $\{F, G\}=-\{G, F\}=\Lambda(d F, d G)$.

ii) $\left\{F_{1},\left\{F_{2}, F_{3}\right\}\right\}+$ cycl. $=0$.

iii) $Y(\{F, G\})=\{Y(F), G\}+\{F, Y(G)\}$.

$4 \quad$ The Lie derivative of by $Y$ shall vanish. We employ this terminology in correspondence with the terminology used for one parameter groups 
The last equation holds for any canonical vectorfield $X$. The vanishing of the cyclic sum in $(2.2$, ii) is equivalent to $\Omega$ being closed. The algebra of differentiable functions on $M$ will be denoted by $\mathfrak{A}$. Any subset $S$ of $\mathfrak{A}$ which is sufficient to define the differentiable structure on $M$ will be called a sufficient set. These sets are the global analogue of a covering by local charts, and play an important role in our discussions. In particular we note that a canonical form may be defined on any suitable manifold by specifying a bracket with $(2.2$, i) and $(2.2$, ii) on any sufficient set, provided the resulting tensorfield $\Lambda$ is nowhere degenerate. The canonical systems we shall study here are required to satisfy the following set of Kinematical Axioms.

Kinematical Axioms: For the canonical system $(M, \Omega, Y)$ there exists a subset $\mathfrak{U}_{0}$ in the algebra $\mathfrak{A}$ of differentiable functions such that:

K.i) $F$ is in $\mathfrak{U}_{0}$ iff $\{F, A\}=0$ for all $A$ in $\mathfrak{A}_{0}$,

K.ii) $\mathfrak{U}_{0} \cap Y\left(\mathfrak{U}_{0}\right)=(0)$,

K.iii) $\mathfrak{U}_{0} \cup Y\left(\mathfrak{U}_{0}\right)$ is a sufficient set,

K.iv) $\{G, A\}$ is in $\mathfrak{U}_{0}$ for $A$ in $\mathfrak{A}_{0}$ and $G=Y(B)$ in $Y\left(\mathfrak{A}_{0}\right)$,

K.v) for any $A$ in $\mathfrak{U}_{0}$ the canonical vectorfield $\xi_{d A}$ generates a group.

By K.iii) we may choose charts $\left(U, A^{i}, Y\left(B^{r}\right)\right)$ at any point in $M$, where $A^{i}$; $i=1, \ldots, n$ and $B^{r} ; r=1, \ldots, m-n$ are functions from $\mathfrak{A}_{0}$. Furthermore we may assume without loss of generality that $B^{r}=b^{r}\left(A^{1}, \ldots, A^{n}\right)$ in $U$. Since the functions $Y\left(B^{r}\right)$ are coordinates in $U$ we conclude that $m-n \leqq n$, and hence $m=2 n$, since the differentials $d A_{q}^{i}$ span at any point $q$ in $U$ an isotropic subspace of the bilinear form defined by $\Lambda$ in the corresponding cotangent space. The Jacobian matrix of the system of functions $\left(b^{r}\right)$ is nonsingular, and we conclude from the implicit function theorem that there exist always special charts of the form $\left(U, B^{s}, Y\left(B^{r}\right)\right)$ at any point in $M$. The functions $\left\{B^{s}, Y\left(B^{r}\right)\right\}$ resp. $\left\{A^{i}, Y\left(B^{r}\right)\right\}$ are in $\mathfrak{A}_{0}$ and form a nonsingular matrix in $U$. The latter matrix may always be chosen to equal unity at some given point in $U$. These facts will frequently be used.

The set $\mathfrak{U}_{0}$ is a subalgebra of $\mathfrak{U}$ which is maximal commutative under the Poisson bracket. This algebra is not uniquely determined by the kinematical axioms.

In fact for any canonical transformation $\varphi$ leaving $Y$ invariant the subalgebra $\varphi\left(\mathfrak{A}_{0}\right)$ will again satisfay all axioms if $\mathfrak{U}_{0}$ does ${ }^{5}$. The functions in $\mathfrak{U}_{0}$ generate by $\left(2.1\right.$, ii) a commutative Lie algebra $\mathscr{L}_{0}$ of canonical vectorfields. Hence we obtain a differentiable, involutive distribution $K$, assigning to every point $p$ a subspace $K_{p}$ of the tangentspace at $p$.

$$
K_{p}=\left(X_{p} \mid X \text { in } \mathscr{L}_{0}\right)
$$

5 A simple example is provided by the manifold $M=R^{2}$ with global coordinates $Q, P$. Choosing $\Omega=d Q \wedge d P$ and $Y=\xi_{d H}$ with $H=P^{2}+Q^{2}$ a canonical system is obtained. For the subalgebra $\mathfrak{A}_{0}$ of functions depending on $Q$ only the kinematical axioms are satisfied. The same holds true if we consider functions of $P$ only, since both coordinates may be permuted by a canonical transformation leaving $H$ invariant 
Any function invariant under $\mathscr{L}_{0}$ is called an integral function $G$ of $K: X(G)=0$ for any $X$ in $\mathscr{L}_{0}$. By K.i) this is true if and only if $G$ is in $\mathfrak{A}_{0}$. Collecting these results we obtain:

Proposition 2.1. The distribution $K$ defined by $\mathfrak{A}_{0}$ is differentiable and involutive. Every subspace $K_{p}$ is maximal isotropic with respect to the bilinear form induced by $\Omega: \operatorname{dim} K_{p}=n$, where $\operatorname{dim} M=2 n$. The algebra of integral functions is equal to $\mathfrak{U}_{0}$.

By the global version of the Frobenius integrability theorem the distribution $K$ defines at any point $p$ in $M$ a unique maximal integral submanifold $p^{*}$ passing through $p$ (see Appendix). Concerning the differentiable structure on any such submanifold we have:

Proposition 2.2. Let $\left(U, A^{i}, Y\left(A^{k}\right)\right)$ be a chart at $p$ on $p^{*}$ with $A^{i}$ in $\mathfrak{I}_{0}$. The functions $Y\left(A^{k}\right), k=1, \ldots, n$ are coordinates everywhere on $p^{*}$. They separate the points on $p^{*}$, and range each over the whole real line on $p^{*}$. Hence $p^{*}$ is diffeomorphic to the vectorspace $R^{n}$.

The proof of Proposition 2.2 makes substantial use of axiom K.v) and may be found in the Appendix. The set of all maximal integral manifolds will be denoted by $M^{*}$. We shall now introduce a topology on $M^{*}$ which is derived from the topology on $M$. For the proof of the following proposition see again the Appendix.

Proposition 2.3. Let $\mathfrak{B}$ be a countable basis of neighbourhoods on $M$. For any $V$ in $\mathfrak{B}$ denote by $V^{*}$ the corresponding subset in $M^{*} ; V^{*}=\left(q^{*} \mid q\right.$ in $\left.V\right)$. Then $\mathfrak{B}^{*}=\left(V^{*} \mid V\right.$ in $\left.\mathfrak{B}\right)$ satisfies all axioms for a basis for neighbourhoods on $M^{*}$. The resulting topological space is a connected Hausdorff space with a countable basis. Any function A in $\mathfrak{U}_{0}$ defines a unique continuous function $A^{*}$ on this space.

It is an immediate consequence of Proposition 2.3 that any point in $M^{*}$ has a neighbourhood which is homeomorphic to an open subset subset of $R^{n}$, $\operatorname{dim} M=2 n$. Furthermore the algebra $\mathfrak{A}_{0}^{*}$ of continuous functions on $M^{*}$ defined by corresponding functions in $\mathfrak{I}_{0}$ defines a differentiable structure on $M^{*}$. In fact by considering the non-singular matrix $\left(\left\{Y\left(A^{i}\right), A^{k}\right\}\right)$ in any arbitrary chart $\left(U, A^{i}, Y\left(A^{k}\right)\right)$ one may show that any function $A$ in $\mathfrak{A}_{0}$ is a function of the coordinates $A^{i}$ alone in $U$.

Using this result it is not difficult to show that the algebra $\mathfrak{A}_{0}^{*}$ satisfies the requirements $(2, \mathrm{i})-(2$, iii) in the Definition 1 . of a manifold given in the Appendix. We have thereby obtained:

Theorem 2.1. Let $(M, \Omega, Y)$ be a canonical system with subalgebra $\mathfrak{A}_{0}$. Denote by $\mathfrak{U}_{0}^{*}$ the corresponding algebra of continuous functions on the space $M^{*}$ of integral submanifolds. Then $\left(M^{*}, \mathfrak{U}_{0}^{*}\right)$ is a manifold, called the quotient of $M$ by $\mathfrak{A}_{0}$. The projection $\pi: M \rightarrow M^{*}$ is a differentiable map.

We mention that a similar though weaker result may be obtained if axiom K.v) is given up. In this case Proposition 2.2 does not hold, and the topological space defined in Proposition 2.3 will not in general be a Hausdorff space. Apart from this, however, the pair $\left(M^{*}, \mathfrak{U}_{0}^{*}\right)$ will still enjoy all other properties which define a manifold. The existence of such more general structures within our framework may be demonstrated by simple examples. For details we refer the reader to [6]. 
We shall now consider functions like $Y(A), A$ in $\mathfrak{U}_{0}$. By K.iv) the corresponding canonical vectorfields define derivations on the subalgebra $\mathfrak{A}_{0}$, and hence on $\mathfrak{U}_{0}^{*}$ as well.

$$
D_{Y(A)}: B^{*} \rightarrow\{Y(A), B\}^{*}, \quad B \quad \text { in } \quad \mathfrak{U}_{0} .
$$

The map $D$ is a map from the set $Y\left(\mathfrak{U}_{0}\right)$ of functions on $M$ to the space $\mathscr{L}\left(M^{*}\right)$ of vectorfields on the quotient $M^{*}$. This map may be extended to the submodule $\mathfrak{U}_{1}$ of functions which are locally linear combinations from $Y\left(\mathfrak{U}_{0}\right)$ with coefficients in $\mathfrak{U}_{0}$. By easy computations in local charts the reader may convince himself that all Kinematical Axioms remain valid if we replace the set $Y\left(\mathfrak{U}_{0}\right)$ by $\mathfrak{A}_{1}$ everywhere. We put $D_{B \cdot Y(A)}=B^{*} \cdot D_{Y(A)}$ and note that this definition is consistent with (2.4).

Proposition 2.4. The map $D$ defined by $D_{G}\left(A^{*}\right)=\{G, A\}^{*}$ for $G$ in $\mathfrak{U}_{1}$ is one-to-one and onto the space of vectorfields on $M^{*}$.

Proof. $D$ is one-to-one by $\mathrm{K}$.ii). Considering charts $\left(U, A^{i}, Y\left(B^{k}\right)\right)$ with matrix $\left(\left\{Y\left(B^{k}\right), A^{i}\right\}\right)$ nonsingular in $U$ we conclude that $D$ induces a mapping onto the space of vectorfields on the open submanifold $U^{*}=\pi(U)$ of $M^{*}$. Covering $M$ with such charts and considering a corresponding decomposition of unity on $M^{*}$ we obtain the result $([8], 1.1$, Theorem 1.3$)$.

By Proposition 2.4 there exists an inverse map for $D$, assigning to any vectorfield $X$ on $M^{*}$ a unique function $P_{X}$ on $M$. We shall call this function the momentum associated to $X$. A corresponding function may be defined on the cotangent bundle $L^{*}$ over $M^{*}$. The elements of $L^{*}$ will be denoted by $\left(q, \omega_{q}\right)$, where $q$ is some point in $M^{*}$ and $\omega_{q}$ is some differential at $q$. For $A^{*}$ in $\mathfrak{U}\left(M^{*}\right)$ and any vectorfield $X$ on $M^{*}$ consider the following functions on $L^{*}$ :

i) $A^{0}\left(q, \omega_{q}\right)=A^{*}(q)$

ii) $P_{X}^{0}\left(q, \omega_{q}\right)=\omega_{q}\left(X_{q}\right)$.

Theorem 2.2. Let $(M, \Omega, Y)$ be a canonical system with quotient $M^{*}$. There exists a unique diffeomorphism $\varphi$ of $M$ with the cotangent bundle $L^{*}$ over its quotient such that:

i) $A=A^{0} \circ \varphi, \quad A$ in $\mathfrak{U}_{0}$;

ii) $P_{X}=P_{X}^{0} \circ \varphi, \quad X$ in $\mathscr{L}\left(M^{*}\right)$.

Proof. Consider the bijective correspondence $\varphi^{*}: A \rightarrow A^{0}, P_{X} \rightarrow P_{X}^{0}$ between the set $S=\mathfrak{U}_{0} \cup \mathfrak{A}_{1}$ and the set $S^{0}$ of all functions as in (2.5). The set $S$ separates the points in $M$ by Proposition 2.2, and the same is true for $S^{0}$. By the same proposition any two corresponding functions range over the same set of values, hence we obtain a unique correspondence $\varphi$ between points in $M$ and in $L^{*}$ such that $\varphi^{*}(F) \circ \varphi=F$ for $F$ in $S$. Since both sets are sufficient $\varphi$ is a diffeomorphism.

The Isomorphism Theorem 2.2 gives a negative answer to a question raised by R. Jost [3] some time ago, which concerned the role played by compact canonical manifolds in mechanics. For a system satisfying the Kinematical Axioms K.i)-K.v) the underlying manifold can never be compact. As a consequence of this theorem 
we may henceforth restrict our attention to canonical systems of the form $\left(L^{*}(N), \Omega, Y\right)$ where $N$ is some manifold (satisfying conditions to be discussed below). We shall classify these systems in the next chapter.

\section{Classification of Canonical Systems}

We begin by a classification of the canonical structures. The notation for functions in $\mathfrak{U}_{0}$ and $\mathfrak{U}_{1}$ will be the same as in Theorem 2.2.

Theorem 3.1. Let $\left(L^{*}(N), \Omega, Y\right)$ be a canonical system. The canonical structure on $L^{*}(N)$ is determined by the following set of relations:

i) $\{A, B\}=0$ for $A^{*}, B^{*}$ in $\mathfrak{U}(N)$;

ii) $\left\{P_{X}, A\right\}^{*}=X\left(A^{*}\right)$ for $X$ in $\mathscr{L}(N), \quad A$ in $\mathfrak{A}(N)$;

iii) $\left\{P_{X}, P_{X_{1}}\right\}=P_{\left[X, X_{1}\right]}+F\left(X, X_{1}\right)$.

$F\left(X, X_{1}\right)$ is in $\mathfrak{U}_{0}$, and defines a closed 2-form $\omega_{F}$ on $N$ by:

iv) $\omega_{F}\left(X, X_{1}\right)=F\left(X, X_{1}\right) *$.

Conversely let $\omega$ be a closed 2-form on $N$. For any two $X, X_{1}$ in $\mathscr{L}(N)$ define $F\left(X, X_{1}\right)$ by iv). Then i), ii), iii) define a unique canonical structure on $L^{*}(N)$.

Proof. For $X, X_{1}$ in $\mathscr{L}(N)$ define $F\left(X, X_{1}\right)$ by iii). By $(2.2$, ii) we have $\{F, A\}=0$ for any $A$ in $\mathfrak{A}_{0}$, hence $F$ is in $\mathfrak{A}_{0}$. From ii) it follows, that the map

$\omega_{F}: X, X_{1} \rightarrow F\left(X, X_{1}\right)^{*}$

is $\mathfrak{U}(N)$-linear and antisymmetric, hence a 2-form. Now for any three vectorfields $X_{1}, X_{2}, X_{3}$ one may calculate the following cyclic sum which vanishes by $(2.2, \mathrm{ii})$ :

$$
\left\{P_{X_{1}},\left\{P_{X_{2}}, P_{X_{3}}\right\}\right\}+\text { cycl. }=0 \text {. }
$$

As a result we obtain:

$$
X_{1}\left(\omega_{F}\left(X_{2}, X_{3}\right)\right)+\omega_{F}\left(X_{1},\left[X_{2}, X_{3}\right]\right)+\text { cycl. }=0 .
$$

Since the left hand side is equal to $d \omega_{F}\left(X_{1}, X_{2}, X_{3}\right)$ the form $\omega_{F}$ is closed.

On the other hand it is not difficult to check that for any closed $\omega_{F}$ the bracket defined by i)-iv) is a derivation as far as the product of variables is defined. The resulting antisymmetric tensorfield $\Lambda$ is nowhere degenerate and defines a Poisson bracket with Equations $(2.2, \mathrm{i})$ and $\left(2.2\right.$, ii) for all variables on $L^{*}(N)$. QED.

For the kinematical vectorfield $Y$ we obtain the following result:

Theorem 3.2. Let $\left(L^{*}(N), \Omega, Y\right)$ be a canonical system. The vectorfield $Y$ defines a nowhere degenerate symmetric tensorfield $g$ on $N$ by:

i) $g\left(d A^{*}, d B^{*}\right)=\{Y(A), B\}^{*} ; \quad A, B$ in $\mathfrak{A}_{0}$.

Denote by $2 T$ the function on $L^{*}(N)$ corresponding to $g$ :

ii) $2 T\left(q, \omega_{q}\right)=g\left(\omega_{q}, \omega_{q}\right)$. 


\section{Then:}

iii) $Y=\xi_{d T}+Y^{0}$,

where

iv) $Y^{0}(A)=0$ for any $A$ in $\mathfrak{U}_{0}$.

Proof. Consider an arbitrary chart $\left(U ;\left(Q^{l}\right)^{*}\right)$ on $N$ and the associated chart $\left(V ; Q^{i}, P_{k}\right)$ on $L^{*}(N): V=\pi^{-1}(U),\left(\left\{P_{k}, Q^{i}\right\}\right)$ equal to the unit matrix on $V$. Then $G^{i k}=\left\{Y\left(Q^{i}\right), Q^{k}\right\}$ is in $\mathfrak{U}_{0}$, and we have $T=\frac{1}{2} \sum_{i k} G^{i k} P_{i} P_{k}$ in $V$.

Defining $Y^{0}$ by iii) we deduce that $\left\{Y^{0}(A), B\right\}=0$ in $V$ for any $A, B$ in $\mathfrak{A}_{0}$, since in $V$ these are functions of the coordinates $Q^{i}$ only. Since $V$ was arbitrary, we have $Y^{0}(A)$ in $\mathfrak{Q}_{0}$. However $Y(A)$ and $\xi_{d T}(A)$ are both in $\mathfrak{A}_{1}$, and hence $Y^{0}(A)=0$.

QED.

Let us illustrate the results obtained in Theorem 3.1 and 3.2 by writing down the corresponding equations in an arbitrary associated chart $\left(V ; Q^{i}, P_{k}\right)$. We may assume that $Y^{0}$ is Hamiltonian in $V: Y_{0}=\xi_{d W}$. Since $Y^{0}(A)=\{W, A\}=0$ for any $A$ in $\mathfrak{A}_{0}$, the function $W$ is a function of the coordinates $Q^{i}$ alone:

$$
Y=\xi_{d H} ; \quad H=\frac{1}{2} \sum_{i k} G^{i k}(Q) P_{i} P_{k}+W(Q) .
$$

For the canonical structure we obtain in $V$ :

i) $\left\{Q^{i}, Q^{k}\right\}=0$,

ii) $\left\{P_{i}, Q^{k}\right\}=\delta_{i}^{k}=1$ for $i=k$ and vanishes otherwise,

iii) $\left\{P_{i}, P_{k}\right\}=F_{i k}(Q)$,

where

$$
X_{i}\left(F_{j k}\right)+\text { cycl. }=0 .
$$

Before we can give a physical interpretation to these equations we have to specify the set of states and the dynamical law. Consider the $C^{*}$-algebra of continuous functions on $L^{*}$ vanishing at infinity. Positive bounded linear functionals on this algebra correspond to measures on $L^{*}([7], I V, \S 20.4)$; let us admit all such functionals as states. If we assume for simplicity that $Y$ generates a group, we define the dynamical law as the corresponding family of maps: $t \rightarrow \sigma_{t}$ from the real line to the set of states:

i) $F \rightarrow F_{t}, \quad d F_{t} / d t=Y\left(F_{t}\right), \quad F \quad$ in $\quad \mathfrak{U}$;

ii) $t \rightarrow \sigma_{t}, \quad \sigma_{t}(F)=\sigma_{0}\left(F_{t}\right)$.

The dynamical law is then additive with respect to the addition of states, which we may hence interpret as describing ensembles of physical systems. From the pure states, which correspond to the points in $L^{*}$ we obtain just the integralcurves of $Y$. Their equations in the given chart are:

$$
\begin{aligned}
& \text { i) } q^{i}(t)=\left(Q^{i}\right)_{t}(p), \quad p_{k}(t)=\left(P_{k}\right)_{t}(p), \quad p \quad \text { in } L^{*} \\
& \text { ii) } d q^{i} / d t=\left\{H, Q^{i}\right\}(p)=\sum_{k=1}^{n} G^{i k}\left(q^{1}, \ldots, q^{n}\right) p_{k} \\
& \text { iii) } d p_{k} / d t=\left\{H, P_{k}\right\}(p)=-\frac{1}{2} \sum_{i j} \partial G^{i j} / \partial q^{k} p_{i} p_{j}-\partial W / \partial q^{k}+\sum_{i j} G^{i j} p_{i} F_{k j} .
\end{aligned}
$$


The theory thus obtained includes in particular the case of non-relativistic particle mechanics, where $M$ is the phase space of some finite number of masspoints. These move in the configuration space $N$ of arbitrary Riemannian structure; external fields and interactions are represented by the potentials $W$ and $F_{k j}$. We may call this the "phase space interpretation" of canonical systems.

However there is a different interpretation which we shall aim at in a following paper. Consider some system of $r$ particles, where the configuration manifold is the $r$-fold product of some fixed single particle position space $N$.

Now consider the subset $\mathfrak{A}^{\prime}$ of variables which are just sums of identical single particle components. We may introduce component-wise multiplication as a composition law within $\mathfrak{I}^{\prime}$, and consider the resulting algebra as our classical algebra of variables. Both the Poisson bracket and $Y$ will map the subset $\mathfrak{I}^{\prime}$ to itself if and only if these decompose into identical components as well:

$$
\Omega=\sum_{a=1}^{r} \Omega_{a}, \quad Y=\sum_{a=1}^{r} Y_{a} .
$$

In this case the vectorfield $Y$ will only describe the motion of particles in external fields. These are coupled in the same way to any single particle, as is apparent from (3.4). However this is just the situation met in relativistic particle physics. As far as the gravitational field is concerned, this fact is expressed by the "principle of equivalence", whereas for the electromagnetic field it is known as the "principle of minimal coupling". The quotient manifold $N$ should then be interpreted as the space-time of general relativity. From this point of view the principles mentioned above do not have to be postulated separately, but result as a necessary consequence if we insist upon a unique space-time interpretation of canonical systems.

In this interpretation states will not describe ensembles but actual distributions of matter. If we wish to describe interactions as mediated by the classical fields, both the canonical structure and the vectorfield $Y$ will themselves have to depend upon the particular state considered. Thus the dynamical law will no longer be additive with respect to the addition of states, the additional terms representing the effects of the interaction. In a subsequent paper [11] we shall develop the theory along these line, concentrating mainly on the structure of $\mathfrak{A}$. The notion of states appropriate to the interpretation sketched here will then be introduced, and a certain simple class of states is shown to describe the motion of relativistic matter in an arbitrary external gravitational and electromagnetic field.

\section{Appendix}

For the definitions and theorems cited here the first chapter of [8] and [9] may serve as a general reference. By differentiable we mean infinitely often differentiable.

Definition 1. A manifold is an ordered pair $(M, \mathfrak{l})$ where

1. $M$ is a connected Hausdorff space having a countable basis of open neighbourhoods.

2. $\mathfrak{A}$ is a set of continuous real-valued functions on $M$ such that

i) for any point $q$ in $M$ there exists a set $\left(Q^{i} ; i=1, \ldots, m\right)$ of functions from $\mathfrak{A}$ defining a homeomorphism of a suitable neighbourhood $U$ of $q$ with an open subset of $R^{m}$; 
ii) on the neighbourhood $U$ any function $F$ in $\mathfrak{U}$ may be written as $F=f\left(Q^{1}, \ldots, Q^{m}\right)$, where $f$ is differentiable;

iii) a given function $G$ on $M$ is in $\mathfrak{A}$ if and only if at any point in $M$ it may be expressed as a differentiable function of finitely many elements from $\mathfrak{U}$.

The functions $Q^{i}$ of (2.1) are called coordinates at $q$; the ordered set $\left(U, Q^{i}\right)$ is called a chart at $q$. The number $m$ of coordinates is independent of $q$ and called the dimension of $M$. Let $M$ be a space satisfying 1 . A set of functions on $M$ satisfying $(2$, i) and $(2$, ii) is called a sufficient set. Given some sufficient set $S$, there exists a unique maximal sufficient set $\mathfrak{U}$ containing $S$, and $(M, \mathfrak{A})$ is a manifold ([8], Chapter I, 1). Most of the notions in differential geometry like differentiable maps, submanifolds, vectorfields may be defined in terms of the algebra $\mathfrak{A}$ or any sufficient set. For a distribution $K$ defined by some Lie algebra of vectorfields on $M$ as in Chapter 2 we call $N$ an integral submanifold, if

$$
\text { 1. } d \varphi_{q}: L_{q}(N) \rightarrow K_{\varphi(q)} \subset L_{\varphi(q)}(M) \text { for any } q \text { in } N \text {, }
$$

where $d \varphi_{q}$ is the differential of the injection $\varphi: N \rightarrow M$, mapping the tangent space $L_{q}(N)$ into the tangent space $L_{\varphi(q)}(M)$.

Integrability Theorem (local version): Let $M$ be a manifold of dimension $m$, and $K$ be a distribution of dimension $m-n$ defined by some Lie algebra of vectorfields. At any point in $M$ there exists a chart $\left(U, F^{1}, \ldots, F^{m}\right)$ such that:

i) the functions $F^{i}, i=1 \ldots n$, are integral functions in $U$, and any integral function $G$ in $U$ may be uniquely expressed as $G=g\left(F^{1}, \ldots, F^{n}\right)$, where $g$ is differentiable;

ii) through any point in $U$ there passes a unique maximal integral submanifold in $U$ defined by an equation $a=\left(a^{1}, \ldots, a^{n}\right)=\left(F^{1}, \ldots, F^{n}\right)$ for a in some open subset $V(U)$ of $R^{n}$;

iii) on any such submanifold I the functions $F^{n+1}, \ldots, F^{m}$ define by restriction $a \operatorname{chart}\left(U \cap I ; F^{n+1}, \ldots, F^{m}\right)$.

Integrability Theorem (global version). Let $M$ be a manifold, and $K$ a distribution on $M$ defined as above. Through any point in $M$ there passes a unique maximal integral submanifold I. Any differentiable map to $M$ which takes its values in $I$ defines a differentiable map to $I$.

For both versions we refer in particular to ([10], Chapter III, §VII, VIII, IX). It is not difficult to see that some function in $\mathfrak{A}$ is an integral function if and only if it is constant on any maximal integral submanifold.

Proof of Proposition 2.2. Let $\left(U, A^{i}, Y\left(A^{k}\right)\right)$ be a chart at $p$. By the local version of the integrability theorem the functions $Y\left(A^{k}\right)$ are coordinates at $p$ on $p^{*}$. It is not difficult to see that the set of functions $\left(A^{i}, Y\left(A^{k}\right)\right)$ is independent if and only if the matrix $\left(\left\{A^{i}, Y\left(A^{k}\right)\right\}\right)_{i k}$ is non-singular. However this matrix has elements constant on $p^{*}$, thus the functions $Y\left(A^{k}\right)$ are coordinates everywhere. Choosing functions $B^{i}, i=1, \ldots, m$ from $\mathfrak{U}_{0}$ such that at $p$ :

$$
\left(\xi_{d B^{i}}\left(Y\left(A^{k}\right)\right)\right)=\left(\left\{B^{i}, Y\left(A^{k}\right)\right\}\right)
$$

equals the unit matrix $\left(\delta^{i k}\right)$ we obtain for the corresponding groups $\phi^{k}$ generated by the fields $\xi_{d B^{k}}$

$$
\phi_{t}^{k}\left(Y\left(A^{i}\right)\right)=Y\left(A^{i}\right)+t \cdot \delta^{i k} \text { on } p^{*}, \quad t \text { in } R .
$$


Finally let $q, p$ be points on $p^{*}$ such that $Y\left(A^{k}\right)(p)=Y\left(A^{k}\right)(q)$ for all $k$. Joining $q$ and $p$ by a curve $\gamma$, we may deforme this curve by means of the groups $\phi^{k}$, such that $Y\left(A^{k}\right)=Y\left(A^{k}\right)(p)$ everywhere on $\gamma$. Hence all tangentvectors defined by $\gamma$ vanish, and $p=q$. QED.

Proof of Proposition 2.3. Let $V^{*}$ and $W^{*}$ contain $p^{*}$. We have to show that $V^{*} \cap W^{*}$ contains some neighbourhood $V_{0}^{*}$ of $p^{*}$. This is obvious if $V$ and $W$ are contained in some common open submanifold $U_{F}$ of $M$, where the local version of the integrability theorem holds (Frobenius chart). If they are not, join any two points $p$ in $V, q$ in $W$ by a curve on $p$. Covering this curve with a finite number of Frobenius charts $U_{F}$, we obtain a finite sequence of open sets $V_{r} ; r=1, \ldots, s$; where $V_{1}=V$, $V_{s}=W$ and any two $V_{r}, V_{r+1}$ are contained in some common $U_{F}$. The result follows by finite induction. The projection $p \rightarrow p^{*}$ of $M$ to $M^{*}$ is continuous, hence $M^{*}$ is connected. Any function $A$ in $\mathfrak{U}_{0}$ is constant on $p^{*}$, hence defines a function $A^{*}$ on $M^{*}$, which is easily seen to be continuous. To show that $M^{*}$ is Hausdorff, assume that $V_{q}^{*} \cap W^{*}=\emptyset$ for any two neighbourhoods of $q^{*}$ and $p^{*}$. It follows that $A^{*}\left(q^{*}\right)=A^{*}\left(p^{*}\right)$ for any $A$ in $\mathfrak{A}_{0}$. Let $\left(U, A^{i}, Y\left(A^{k}\right)\right)$ be a Frobenius chart in $V_{q}$ at $q$. The functions $Y\left(A^{k}\right)$ are coordinates on $p^{*}$, and there exists a unique point $p$ on $p^{*}$ such that $Y\left(A^{k}\right)(q)=Y\left(A^{k}\right)(p)$ for all $k$. Choosing an arbitrary Frobenius chart $\left(W, A^{i}, Y\left(A^{k}\right)\right)$ at $p$, we can find an integral manifold $p_{1}^{*}$ passing through both $U$ and $W$. This manifold contains a unique point $p_{1}$ such that $Y\left(A^{k}\right)\left(p_{1}\right)=Y\left(A^{k}\right)(q)$, which is hence contained in both $U$ and $W$. Since $M$ is Hausdorff, we have $q=p$, and hence $q^{*}=p^{*}$. QED.

\section{References}

1. Segal,I.E.: Postulates for general quantum mechanics. Ann. Math. 48, 930-948 (1947)

2. Haag, R., Kastler, D.: An algebraic approach to quantum field theory. J. Math. Phys. 5, 848-861 (1964)

3. Jost, R.: Poisson brackets (An unpedagogical lecture). Rev. Mod. Phys. 36, 572-579 (1964)

4. Ehlers, J., Schild, A.: Geometry is a manifold with projective structure, Commun. math. Phys. 32, 119-146 (1973)

Ehlers, J., Pirani, F.A.W., Schild, A.: General relativity, papers in honour of J.L. Synge (ed. L.O'Raifeartaigh) 63-84. Oxford: Clarendon Press 1972

5. Sniatycki, J., Tulczyjew, W. M.: Canonical dynamics of relativistic charged particles. Ann. Inst. H. Poincaré A, 15, 177-187 (1971)

Kijowski,J.: A finite-dimensional canonical formulation in the classical field theory. Commun. math. Phys. 30, 99-128 (1973)

6. Drühl,K.: Relativistic canonical systems, preprint Max-Planck-Institut zur Erforschung der Lebensbedingungen. Starnberg 1975

7. Neumark, M.A.: Normierte Algebren. Berlin: VEB Deutscher Verlag der Wissenschaften 1959

8. Helgason, S.: Differential geometry and symmetric spaces. New York, London: Academic Press 1962

9. Hermann, R.: Differential geometry and the calculus of variations. New York, London: Academic Press 1968

10. Chevally, C.: Theory of Lie groups, 6th printing 1965. Princeton: Princeton University Press 1946

11. Drühl, K.: On the space-time interpretation of classical canonical systems. II: Relativistic canonical systems. Commun. math. Phys. 49, 289-300 (1976)

Communicated by J. Ehlers

Received: June 2, 1975 